\title{
Phorbol Esters Potentiate Evoked and Spontaneous Release by Different Presynaptic Mechanisms
}

\author{
Jack Waters and Stephen J Smith \\ Department of Molecular and Cellular Physiology, Beckman Center, Stanford Medical School, Stanford, California 94305
}

\begin{abstract}
Phorbol esters enhance release from a variety of cell types. The mechanism by which phorbol esters potentiate presynaptic release from central neurons is unclear, although effects of phorbol esters both on the readily releasable pool of vesicles and on presynaptic calcium channels have been shown. Using confocal microscopy and the fluorescent styryl dye FM 1-43, we have examined the effects of phorbol-12,13-dibutyrate (PDBu) on presynaptic vesicle turnover at individually identified synapses in dissociated cultures obtained from neonatal rat hippocampus. Using different dye staining and destaining protocols we were able to resolve two effects of PDBu. Potentiation of evoked release by PDBu was insensitive to calcium channel antagonists, suggesting that this effect results from an increased number of vesicles in the readily releasable pool. Since we observed no effect of PDBu on the size of the total recycling vesicle pool, we
\end{abstract}

Phorbol ester treatment enhances exocytosis from many excitable and nonexcitable cell types. Several mechanisms have been suggested, including (1) an increase in the size of the readily releasable pool of vesicles (Gillis et al., 1996; Stevens and Sullivan, 1998), (2) a decreased rate of calcium clearance from the cytoplasm (Lin et al., 1994), (3) increased calcium influx through an effect on calcium channels (Stea et al., 1995), and (4) increased calcium influx by broadening the action potential through inhibition of potassium channels (Hoffman and Johnston, 1998).

Gillis et al. (1996) presented strong evidence that phorbols increase release from chromaffin cells by increasing the number of vesicles in the readily releasable pool. Altered calcium influx and clearance were not responsible, and phorbols did not affect the calcium sensitivity of release. These conclusions are consistent with those of Vitale et al. (1995), who observed disruption of the cortical actin ring and an increased number of docked vesicles after exposure of chromaffin cells to phorbol ester.

Release from neuroendocrine cells differs from synaptic transmission in several important respects, but studies of neuronal preparations have, likewise, implicated the readily releasable pool. Redman et al. (1997) reported phorbol-induced potentiation at the frog neuromuscular junction despite decreased presynaptic calcium influx. At hippocampal synapses, phorbol esters potentiate release in response to both electrical stimulation and application of hypertonic solutions (Malenka et al., 1996; Stevens and Sullivan, 1998). Because hypertonic solutions cause release in a calciumindependent manner, these data again suggest that phorbol esters potentiate release principally though an effect on the readily releasable pool.

In contrast, some authors have concluded that phorbols act via an increase in calcium channel activity. Parfitt and Madison (1993) found that phorbols increased the frequency of spontaneous min-

Received May 15, 2000; revised July 24, 2000; accepted July 27, 2000.

This work was supported by National Institute of Mental Health Silvio Conte Centre for Neuroscience Research Grant MH48108 to S.J.S. We thank Ron Holz, Murali Prakriya, and Stephen M Smith for critical reading of this manuscript.

Correspondence should be addressed to Jack Waters at the above address. E-mail: jwaters@leland.stanford.edu.

Copyright (C) 2000 Society for Neuroscience $0270-6474 / 00 / 207863-08 \$ 15.00 / 0$ conclude that phorbol esters alter the equilibrium between reserve and readily releasable pools. An additional effect of PDBu on spontaneous release was observed. This effect was antagonized by nifedipine but not $\omega$-conotoxin GVIA or $\omega$-agatoxin IVA. We conclude that PDBu influences spontaneous and evoked release by two different mechanisms: through L-type calcium channels and through an increase in the proportion of recycling vesicles in the readily releasable pool. In addition to further clarifying the mechanism of action of phorbol esters, these results suggest that phorbol esters may be a useful tool with which to probe the function of the readily releasable pool of presynaptic vesicles at CNS synapses.

Key words: hippocampus; neuron; L-type calcium channel; readily releasable pool; vesicle; FM 1-43; protein kinase; release probability

iature EPSPs through activation of L-type calcium channels at hippocampal terminals. Inhibition of N-type channels, which are partially responsible for evoked release, had no effect on the increase in spontaneous frequency induced by phorbols (Parfitt and Madison, 1993). However, L-type channels are not involved in evoked release from hippocampal synapses (Parfitt and Madison, 1993; Wheeler et al., 1994), and the effects of calcium channel antagonists on phorbol-induced potentiation of evoked release are unknown. Whether potentiation of evoked release by phorbol esters is partially attributable to effects on calcium channels is therefore unclear.

We have used established optical techniques to investigate the effects of phorbol esters on synaptic release from hippocampal terminals, distinguishing between effects on evoked and spontaneous release. Like Parfitt and Madison (1993), we find that phorbols increase spontaneous release after calcium influx through L-type calcium channels. However, we find that potentiation of action potential-evoked release is not sensitive to calcium channel antagonists and does not result from a change in the total number of recycling vesicles. We conclude that phorbol esters influence spontaneous and evoked release from hippocampal neurons by two different mechanisms: through a mechanism involving L-type calcium channels and through an increase in the proportion of presynaptic vesicles in the readily releasable pool.

\section{MATERIALS AND METHODS}

Preparation of dissociated cultures. Dissociated hippocampal cultures were prepared from postnatal day 2 Sprague Dawley rats. Hippocampi were dissected, and the dentate gyrus was removed. After treating for $15 \mathrm{~min}$ at room temperature in $10 \mathrm{mg} / \mathrm{ml}$ trypsin, the tissue was dissociated by trituration through the tip of a fire-polished, siliconized glass Pasteur pipette. Dissociated cells were collected by centrifugation at $800 \times g$ at $4^{\circ} \mathrm{C}$ and plated onto Matrigel-coated coverslips in Neurobasal medium (Life Technologies, Gaithersburg, MD) supplemented with B-27 (Life Technologies), $28 \mathrm{~mm}$ glucose, $1.3 \mu \mathrm{M}$ transferrin (Calbiochem, La Jolla, CA), 2 $\mathrm{mm}$ glutamine, $0.7 \mathrm{U} / \mathrm{ml}$ insulin (Sigma, St. Louis, MO), and $1 \%$ fetal calf serum (Hyclone, Logan, UT). Cells were maintained at $37^{\circ} \mathrm{C}$ in an atmosphere containing $5 \% \mathrm{CO}_{2}$ until use after $10-16 \mathrm{~d}$ in vitro.

FM 1-43 staining and destaining. A coverslip was mounted in a custommade, low-volume $(60 \mu \mathrm{l})$ laminar perfusion chamber on the stage of an inverted microscope (IM 35; Zeiss, Thornwood, NY). This permitted continuous perfusion at $\sim 1 \mathrm{ml} / \mathrm{min}$ while imaging the cells using either 
Figure 1. Example of FM 1-43 staining. $a$, Nomarski image of a pyramidal neuron in a mixed neuronal-glial dissociated culture. $b$, Fluorescence image of the same field acquired after FM 1-43 staining, using a 100 -stimulus train at $10 \mathrm{~Hz}$ and a $40 \mathrm{sec}$ exposure to FM 1-43. Discrete fluorescent puncta are visible, often at sites corresponding to dendritic interactions visible in the Nomarski image. $c$, Fluorescence image acquired after destaining using a 900 -stimulus train at $10 \mathrm{~Hz}$. Note that the punctate staining is primarily absent, leaving a dim background attributable to nonspecific membranous staining by FM 1-43. Scale bar, $10 \mu \mathrm{m}$. (a) Nomarski

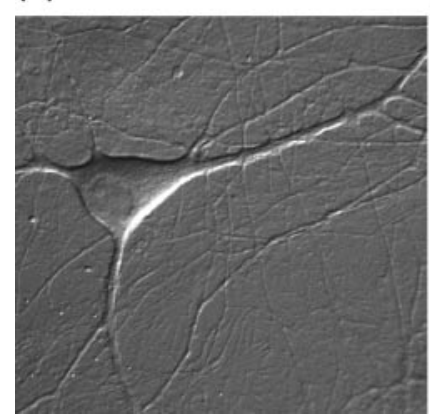

(b) Stained

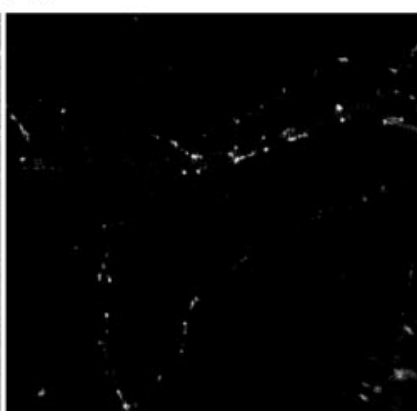

(c) Destained

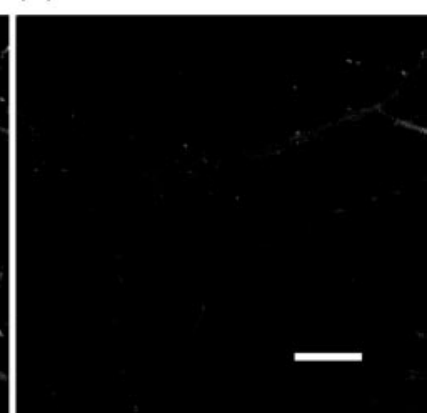

transmitted light and Nomarski optics or in an epifluorescence configuration through the coverslip to which the cells adhered. Cells were perfused with a modified Tyrodes solution consisting of (in $\mathrm{mM}$ ): $119 \mathrm{NaCl}, 5 \mathrm{KCl}$, $2 \mathrm{CaCl}_{2}, 2 \mathrm{MoCl}_{2}, 25 \mathrm{HEPES}$, and 30 glucose, with (in $\left.\mu \mathrm{M}\right) 10 \mathrm{CNQX}, 50$ APV, and 3 bicuculline added to reduce spontaneous and recurrent activity. All imaging experiments were performed at room temperature $\left(22-23^{\circ} \mathrm{C}\right)$.

Stock solution of FM 1-43 at $3 \mathrm{~mm}$ in water was stored at $4^{\circ} \mathrm{C}$. This was diluted into Tyrodes solution to a final concentration of $15 \mu \mathrm{M}$. Timing of the addition of FM 1-43 to the perfusing solution and the duration of stimulation depended on the experimental protocol used. Exocytosis was induced using trains of $1 \mathrm{msec}$ field stimuli at $50 \mathrm{~V} / \mathrm{cm}$ delivered through platinum electrodes positioned on opposite sides of the perfusion chamber. Control experiments using the intracellular calcium dye fluo-4 AM to detect action potential-induced calcium transients verified that stimulation invariably succeeded in firing neurons at the required frequency. After staining, the preparation was washed in dye-free medium for $10 \mathrm{~min}$ to reduce nonspecific staining before image acquisition. Subsequent stimulation resulted in destaining of the preparation.

Stock solutions of phorbol-12,13-dibutyrate $(\mathrm{PDBu})$ were made at $1 \mathrm{~mm}$ in DMSO and stored at $-20^{\circ} \mathrm{C}$ until use. PDBu was added to the perfusing Tyrodes solution to a final concentration of $1 \mu \mathrm{M}$. The resulting concentration of DMSO $(0.1 \%, \mathrm{v} / \mathrm{v})$ did not influence FM 1-43 staining or destaining in control experiments.

Imaging techniques. The sample was illuminated using the $488 \mathrm{~nm}$ line of an air-cooled argon ion laser at the minimal intensity commensurate with an acceptable signal-to-noise ratio of the fluorescent dye $(60 \mu \mathrm{W}$ at the back aperture of the objective). Laser light was focused onto the preparation using an oil immersion objective lens $(40 \times, 1.3$ numerical aperture; DApo UV; Olympus Optical, Tokyo, Japan), and emitted fluorescence was collected through an OG $520 \mathrm{~nm}$ long-pass filter. A Bio-Rad (Hercules, CA) MRC 500 confocal laser scanning microscope running dedicated software with custom modifications was used to acquire images. Images were stored digitally for off-line analysis using custom software (View; Dr. Noam Ziv, Rappaport Institute, Haifa, Israel) or a commercial equivalent (Metamorph; Universal Imaging, West Chester, PA). Five images were acquired after each staining or destaining step, and average fluorescence intensities were calculated offline. Fluorescence intensity measurements were taken from regions of interest of $\sim 1.5 \times 1.5 \mu \mathrm{m}$, corresponding to individual puncta, each visibly separate from its nearest neighbors. Regions of staining $>1.5 \mu \mathrm{m}$ in diameter were excluded from analysis. All fluorescence intensities were corrected for nonspecific staining by subtracting the intensities measured after complete destaining of the preparation.

\section{RESULTS}

The fluorescent styryl dye FM 1-43 has proven a valuable tool with which one can monitor exocytosis and endocytosis of vesicles at presynaptic terminals (Murthy, 1999). When applied to neuronal preparations such as that shown in Figure $1 a$, dye is trapped in endocytosed vesicles after synaptic activity (Betz and Bewick, 1992; Ryan et al., 1993; Betz et al., 1996). After removal of extracellular dye, a punctate staining pattern is observed (Fig. $1 b$; Henkel et al., 1996). Fluorescence intensity may be used as a quantitative measure of the number of vesicles endocytosed at each site of synaptic recycling (Ryan et al., 1997). FM 1-43 associates reversibly with the bilayer and therefore is released on subsequent exocytosis of labeled vesicles (Fig. 1c). Hence, after staining and subsequent removal of extracellular dye, the loss of fluorescence during a subsequent stimulus can be used to monitor vesicle release.

\section{PDBu potentiates FM 1-43 staining}

We have examined the effect of $1 \mu \mathrm{M}$ PDBu on FM 1-43 staining induced by brief stimulus trains consisting of 30 stimuli at $20 \mathrm{~Hz}$.
This stimulus train will turn over the readily releasable pool of synaptic vesicles (Murthy and Stevens, 1998). The protocol used is summarized in Figure 2a. After perfusion of FM 1-43 onto the preparation, a stimulus train consisting of 30 stimuli at $20 \mathrm{~Hz}$ was delivered to induce synaptic vesicle turnover. FM 1-43 was removed from the perfusion chamber 1 min later. After a $10 \mathrm{~min}$ wash period to reduce nonspecific staining, five images were acquired (Fig. $2 a$, images labeled $a$ ) and averaged offline. The fluorescence intensities of puncta in these images represent the number of vesicles endocytosed during the 1 min period in FM 1-43. Fluorescence was then released using a 900 stimulus train at $10 \mathrm{~Hz}$ (sufficient to turn over the entire vesicle pool; Ryan and Smith, 1995), and five images representing nonspecific staining were acquired (Fig. $2 a$, images labeled $b$ ). We will refer to each round of staining and subsequent destaining as one "trial." After a $10 \mathrm{~min}$ rest period and an additional $5 \mathrm{~min}$ in $1 \mu \mathrm{M}$ PDBu, a second, identical trial was conducted. Using this protocol, we compared vesicle turnover at identified synapses before and after exposure to $\mathrm{PDBu}$. Control experiments were conducted in which both trials were performed without PDBu treatment.

The fluorescence intensities of puncta during consecutive trials are plotted in Figure $2 b$. Each point represents the intensities of a single fluorescent punctum corrected for nonspecific staining, i.e., $b-a$ (see Fig. 2a) for the first trial and $c-d$ for the second. Control and PDBu treatment data sets were derived from sister cultures.

These data are presented as frequency histograms in Figure $2 c$. Control synapses stained equally with FM 1-43 during the first and second trials (Fig. 2c, top panel). In contrast, exposure to $\mathrm{PDBu}$ between trials resulted in a pronounced increase in dye staining during the second trial (Fig. $2 b$, bottom panel; $p<0.0001$, Wilcoxon signed rank test). The effect of $\mathrm{PDBu}$, expressed as a percentage change in FM 1-43 loading, is plotted as a frequency histogram in Figure $2 d$. The data are positively skewed. The median effect was a $172 \%$ enhancement of dye staining ( $n=1003$ puncta; mean enhancement, $244 \%$ ), compared with a $0.3 \%$ (median) loss of fluorescence staining in controls $(n=230$ puncta). Potentiation by $\mathrm{PDBu}$ was observed throughout numerous culture preparations.

These data indicate that PDBu potentiated release. A brief train (30 stimuli at $20 \mathrm{~Hz}$ ) was used to stain synaptic vesicles, because a train of this duration provides an estimate of the size of the readily releasable pool (Murthy and Stevens, 1998). Hence, the observed effect of PDBu probably resulted from an increase in the number of vesicles in the readily releasable pool, although other effects of $\mathrm{PDBu}$ cannot be excluded at this point. The possible mechanisms of action of PDBu include (1) mobilization of new vesicles to the readily releasable pool, (2) a redistribution of existing recycling vesicles into the readily releasable pool, (3) an increase in the rate of spontaneous release, and (4) an increased probability of release per vesicle, without an alteration in the number of readily releasable vesicles. These possibilities are considered in turn below.

\section{PDBu does not alter total pool size}

One possible mechanism of action of phorbol esters might be to increase the total number of vesicles available for release, termed 
(a)

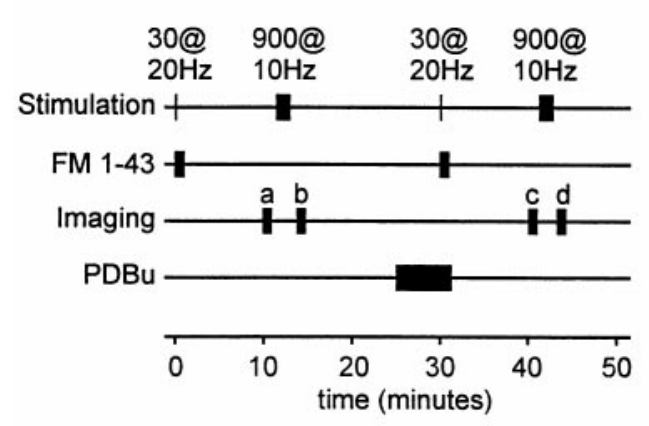

(b)

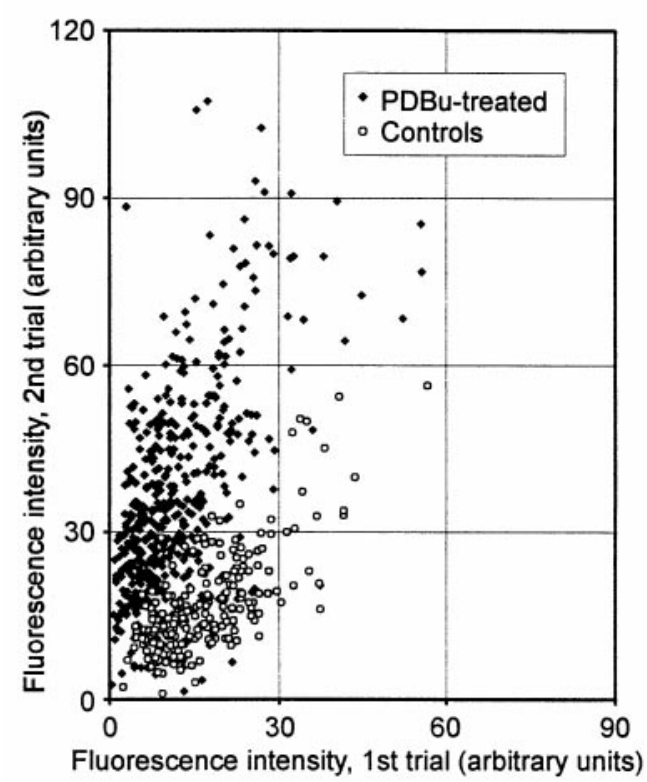

(c)
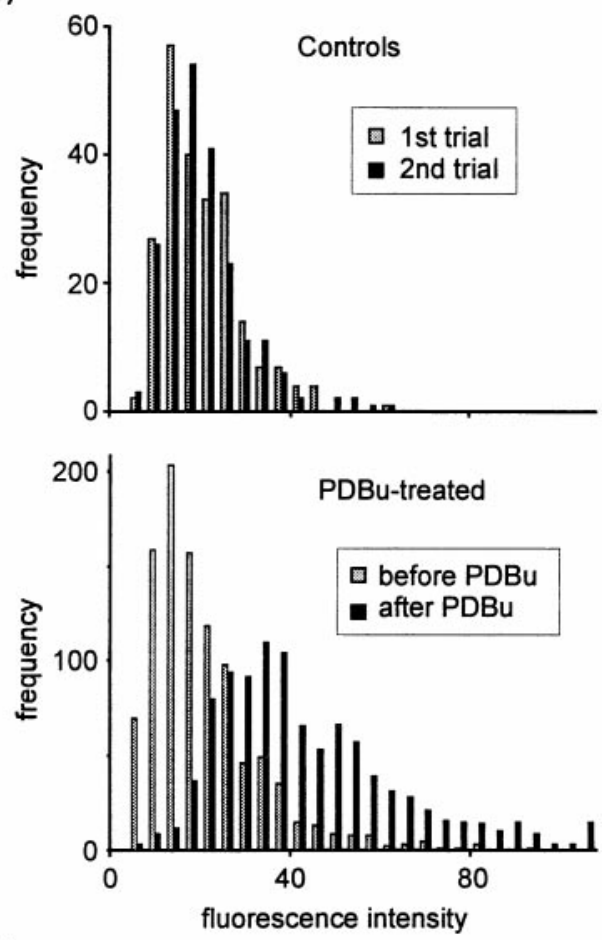

(d)

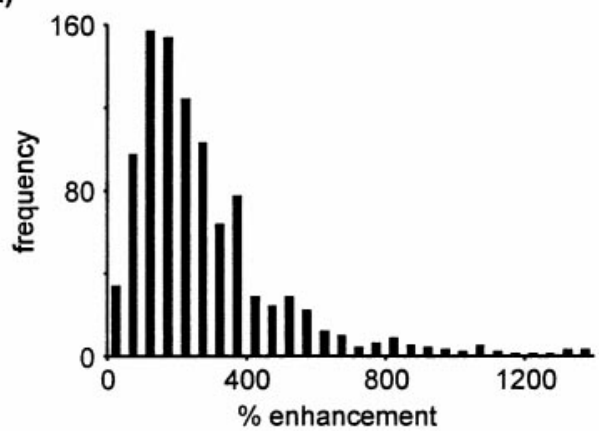

Figure 2. Effect of PDBu on staining. $a$, Summary of the staining-destaining protocol used. $b$, Scatter plot showing fluorescence intensities for individual fluorescent puncta in two consecutive trials. $\mathrm{PDBu}$ resulted in increased FM 1-43 staining ( filled symbols), whereas controls (open symbols) displayed no mean change in staining. $c$, Data represented as frequency histograms. Top panel, control data; bottom panel, effect of PDBu treatment. $d$, Frequency histogram showing the percentage increase in staining observed after PDBu exposure. the total recycling pool. The readily releasable and reserve pools of presynaptic vesicles (which together constitute the total recycling pool) are in dynamic equilibrium, with exchange occurring with a time constant of a few minutes (Ryan and Smith, 1995; Murthy and Stevens, 1999). The addition of vesicles to the reserve pool might therefore increase the size of the readily releasable pool, accounting for the effect of PDBu reported above.

The size of the total recycling pool is easily determined by labeling all the vesicles in a synapse with FM 1-43. To this end we repeated the above experiments using a different loading protocol. Preparations were exposed to 900 stimuli at $10 \mathrm{~Hz}$ during perfusion with FM 1-43. Dye was removed from the perfusion chamber $1 \mathrm{~min}$ after cessation of the stimulus train. This procedure ensures that all available synaptic vesicles are exocytosed and labeled with dye (Ryan and Smith, 1995; Ryan et al, 1996). Destaining was performed using another 900-stimulus train at $10 \mathrm{~Hz}$. As before, the protocol was repeated after a $10 \mathrm{~min}$ wash period and a $5 \mathrm{~min}$ exposure to $\mathrm{PDBu}$. This protocol is illustrated schematically in Figure $3 a$. The fluorescence intensities of each punctum during the two trials are plotted in Figure $3 b$, and the data are represented as frequency histograms in Figure $3 c$.

Using this protocol, no potentiation by phorbol ester was observed ( $n=154$ puncta), indicating that the effect of PDBu on staining in response to brief stimuli does not reflect an increase in the total number of vesicles available for release. [No change in dye staining was observed in control experiments without PDBu treatment (data not shown).] The above data exclude mobilization of new vesicles as a mechanism of action of PDBu.

\section{Effect of PDBu on spontaneous release}

The brief staining protocol described above (Fig. $2 a$ ) was designed to estimate the size of the readily releasable pool. However, in this protocol, synaptic terminals are exposed to FM 1-43 for 1 min. If spontaneous release occurs with sufficient frequency during this period, spontaneously released vesicles may contribute to the observed fluorescence staining. Since phorbol treatment increases spontaneous release from hippocampal terminals (Parfitt and Madison, 1993), this could potentially account for the observed effects of PDBu.

To determine whether spontaneous release is strongly affected by PDBu, we repeated the staining protocol (see Fig. $2 a$ ) with the exclusion of the 30 -stimulus train (Fig. 4a). This protocol measures the amount of spontaneous release occurring during the $1 \mathrm{~min}$ exposure to FM 1-43, in the absence of evoked release. (Note that a third round of staining and destaining was used to locate synapses regardless of their initial rates of spontaneous release; see legend to Fig. 4a.) After phorbol treatment, spontaneous release was greatly increased. This is illustrated for individual fluorescent puncta in Figure $4 b$ and as a frequency histogram in Figure $4 c$. The median increase in fluorescence was $920 \%(n=194$; mean increase, $3230 \%$ ).

\section{Comparison of spontaneous and evoked staining}

Careful comparison of the above data derived from staining protocols with and without evoked release reveals that spontaneous release accounts for only part of the effect of PDBu observed using the staining protocol described in Figure $2 a$. 


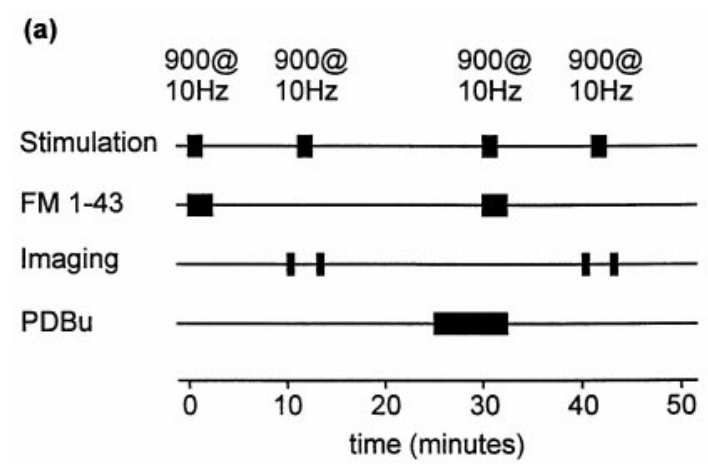

(b)

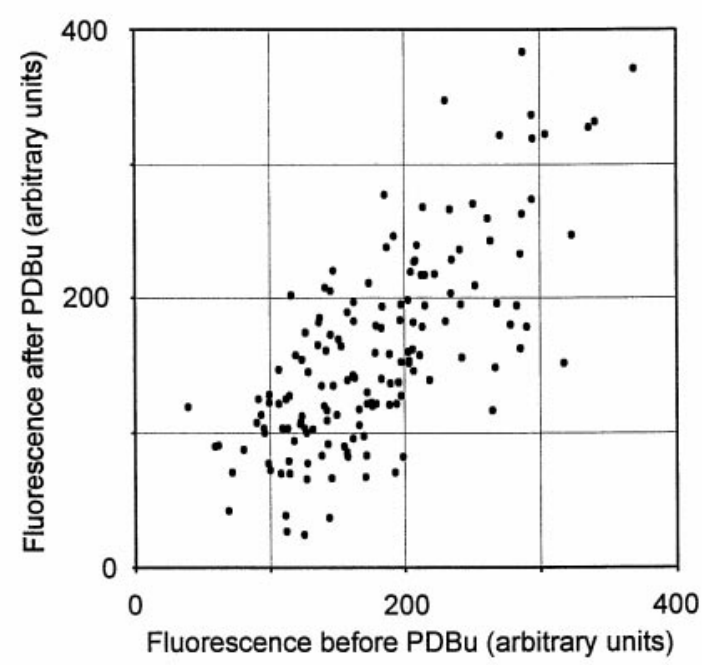

(c)

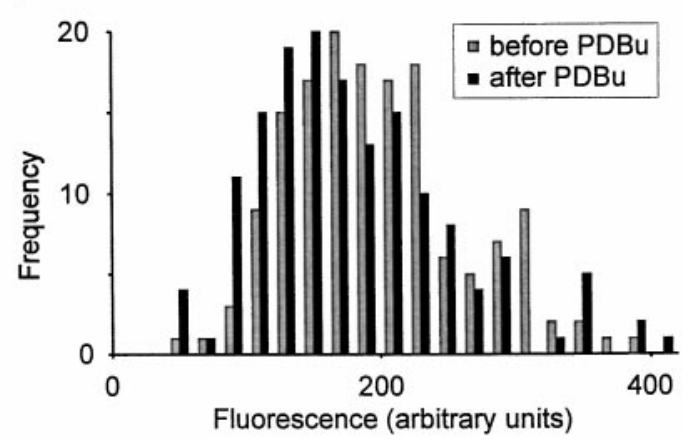

Figure 3. PDBu and total recycling pool size. $a$, Schematic illustration of the protocol used to examine the effect of phorbol treatment on total recycling pool size. $b$, Scatter plot comparing fluorescence staining before and after PDBu treatment. $c$, Data represented as a frequency histogram.

Median fluorescence intensities after spontaneous releaseinduced staining were 0.84 before and 15.1 after PDBu treatment (arbitrary fluorescence intensity units). Median intensities for the staining protocol (using 30 stimuli at $20 \mathrm{~Hz}$ and 1 min in FM 1-43) were 13.8 before and 34.7 after PDBu treatment. These effects of $\mathrm{PDBu}$ on spontaneous and evoked release are summarized in Figure 5.

Since the fluorescence measured using the staining protocol is the sum of spontaneous and evoked release, one can calculate the amount of staining that is attributable to evoked release by subtracting the contribution of spontaneous release from the fluorescence observed using the staining protocol. Hence, evoked release accounts for 13.0 fluorescence units before and 19.3 units after $\mathrm{PDBu}$ treatment. PDBu therefore increased evoked staining from 13.0 to 19.3 arbitrary fluorescence units, a $49 \%$ increase.

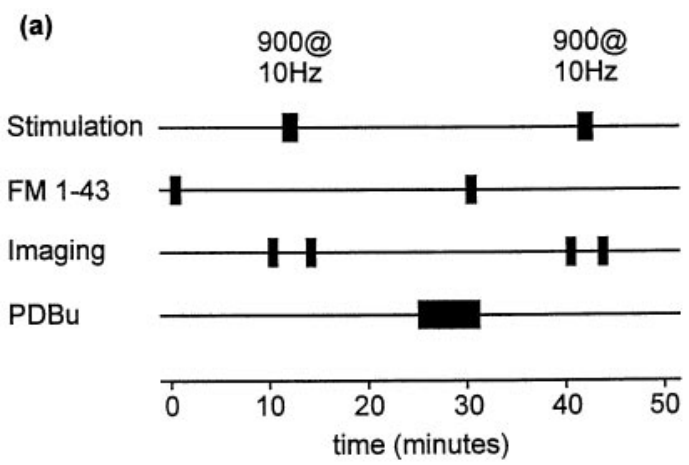

(b)

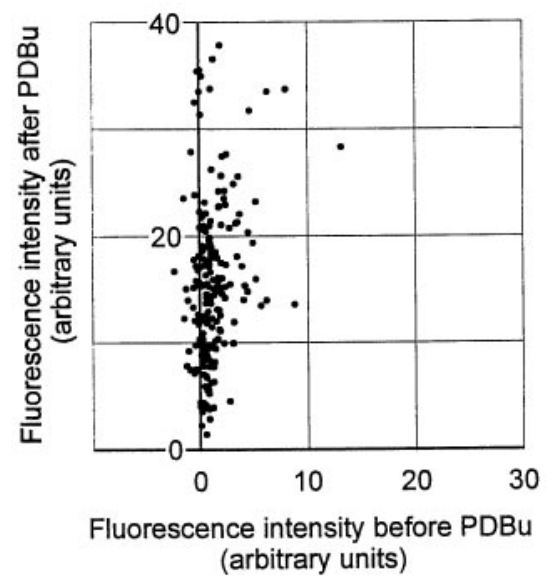

(c)

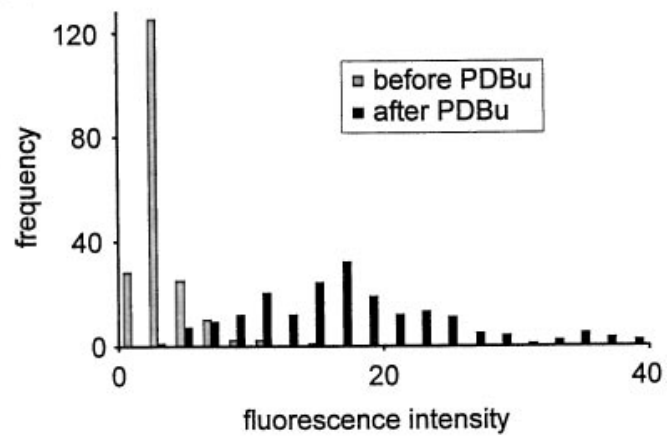

Figure 4. Strong effect of PDBu on spontaneous release. $a$, Protocol used to measure spontaneous release occurring during 1 min. FM 1-43 was applied for $1 \mathrm{~min}$ in the absence of stimulation. As in other protocols, destaining was performed using a 900-stimulus train at $10 \mathrm{~Hz}$. For these experiments a third trial was also performed in which vesicles were stained using a 100-stimulus train at $10 \mathrm{~Hz}$ (trial not shown). The data from this third trial were used verify that the sites of fluorescence staining were positionally stable. This was necessary during these experiments, because many puncta exhibited very weak loading during the first trial, raising the possibility that a fluorescent punctum that was visible only during the second trial represented mobile fluorescence rather than a site at which recycling had been promoted by PDBu treatment. This precaution should therefore exclude the possibility that our selection procedure favored PDBu-sensitive synapses. $b$, Scatter plot showing fluorescence intensities before and after PDBu treatment. $c$, Data presented as a frequency histogram.

These calculations indicate that an increased rate of spontaneous release accounts for part of the effect of PDBu. However, PDBu also influences evoked release (by altering the proportion of recycling vesicles in the readily releasable pool and/or the release probabilities of individual readily releasable vesicles). Were it possible to measure the effect of PDBu on evoked release alone, one would expect an increase in release of $\sim 50 \%$. 


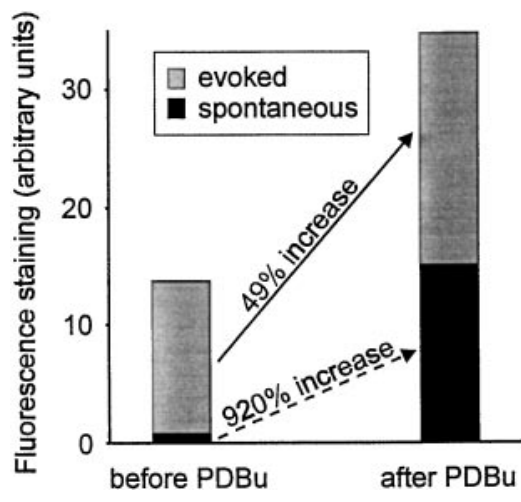

Figure 5. Summary of PDBu effect. Schematic showing the increase in fluorescence staining ( 30 stimuli at $20 \mathrm{~Hz}, 1 \mathrm{~min}$ in FM 1-43) before and after PDBu treatment. The total height of each bar represents the observed fluorescence. Gray and black portions indicate the respective contributions of evoked and spontaneous release. Data are derived from Figures 2 and 5. PDBu potentiates spontaneous release by $920 \%$ and evoked release by $49 \%$. The result is that the contribution of spontaneous release to total staining is much greater after phorbol treatment.

\section{Effect of PDBu on FM 1-43 destaining}

Using a destaining protocol designed to minimize the influence of spontaneous release, we have measured the effect of $\mathrm{PDBu}$ on evoked release and compared the result with the 50\% increase predicted from these calculations.

We began by staining vesicles with a more prolonged stimulus train consisting of 100 stimuli at $10 \mathrm{~Hz}$. This protocol results in the release of $\sim 50 \%$ of the available vesicles at each synapse (Ryan and Smith, 1995). FM 1-43 was removed from the perfusing solution 30 sec after cessation of the stimulus train, by which time $\sim 60 \%$ of released vesicles are labeled (Ryan et al., 1995). The preparation was then washed in dye-free solution for $10 \mathrm{~min}$, sufficient time for dye-labeled vesicles to partition equally between reserve and readily releasable pools (Ryan and Smith, 1995; Murthy and Stevens, 1999). Five fluorescence images were acquired (Fig. 6a, images labeled $a$ ), and then the readily releasable pool was exocytosed using 30 stimuli at $20 \mathrm{~Hz}$. After further image acquisition (Fig. $6 a$, images labeled $b$ ), all remaining vesicles were released using a 900 -stimulus train at $10 \mathrm{~Hz}$, and then more images were acquired (Fig. $6 a$, images labeled $c$ ). To measure the percentage of vesicles released by 30 stimuli after $\mathrm{PDBu}$ treatment, another identical trial then was conducted. The preparation was treated with PDBu for $5 \mathrm{~min}$, beginning $5 \mathrm{~min}$ after dye staining (i.e., after FM 1-43 was removed from the perfusion chamber). The 30stimulus destaining train was delivered in the presence of PDBu during this second trial. This protocol is summarized in Figure $6 a$. It is important to note that this protocol, in which PDBu is applied after the second round of staining, ensured that dye uptake was not influenced by $\mathrm{PDBu}$ treatment.

The effect of PDBu is illustrated in Figure $6 b$. The data are presented as the percentage of vesicles released by 30 stimuli at 20 $\mathrm{Hz}$ during each trial. The percentage of vesicles released during each trial was calculated using mean fluorescence intensities manipulated in the following manner: first trial, percent released by 30 at $20 \mathrm{~Hz}=100 *((a-b) /(a-c))$; second trial, percent released by 30 at $20 \mathrm{~Hz}=100 *((d-e) /(d-f))$, where $a-f$ represent mean fluorescence intensities of the five images acquired at time points indicated in Figure $6 a$.

These data are plotted as a frequency histogram in Figure $6 c$. A significant effect of PDBu was observed $(n=772 ; p<0.0001$, Wilcoxon signed rank test). No effect was observed in control experiments in which $\mathrm{PDBu}$ treatment was excluded (data not shown). A frequency histogram showing the effect of phorbol ester, expressed as a percentage increase in the proportion of fluorescence released following PDBu treatment, is illustrated in Figure $6 d$. The data display a positive skew.

The median increase in evoked release by PDBu was 52\% (mean increase, $140 \%$ ). This figure is very similar to the $49 \%$ increase predicted above and strongly supports the assertion that the destaining protocol measures evoked release and the staining protocol measures a mixture of spontaneous and evoked release.

\section{Effects of calcium channel antagonists}

The above data suggest that phorbol esters enhance release by altering the proportion of recycling vesicles released by a brief stimulus train. Since phorbol esters increase release in response to hypertonic solutions (Stevens and Sullivan, 1998), this probably reflects a redistribution of vesicles from the reserve to the readily releasable pool. However, $\mathrm{PDBu}$ may act to increase release probability, perhaps by increasing calcium entry through voltage-gated calcium channels (Stea et al., 1995). In addition, the potentiating effect of phorbol esters on spontaneous release has previously been shown to require L-type calcium channel activity (Parfitt and Madison, 1993). To determine whether the effects reported above were the result of potentiation of presynaptic calcium currents by $\mathrm{PDBu}$, we therefore examined the effects of PDBu on both evoked and spontaneous release in the presence of calcium channel antagonists.

We began by examining the roles of calcium channel subtypes in evoked release (without PDBu treatment). A total of $10 \mu \mathrm{M}$ nifedipine had no significant effect on release (Fig. $7 a ; p=0.38$, Wilcoxon signed rank test). In contrast, both $1 \mu \mathrm{M} \omega$-conotoxin GVIA $(\omega$-CTx-GVIA) and $500 \mathrm{~nm} \omega$-agatoxin IVA ( $\omega$-Aga-IVA) significantly inhibited evoked release (Fig. $7 a$; each $p<0.0001$, Wilcoxon signed rank test). These concentrations of antagonists are sufficient to selectively eliminate $\mathrm{L}-, \mathrm{N}-$, and $\mathrm{P} / \mathrm{Q}$ - type calcium channels, respectively, and the results are consistent with previous studies, which indicated that release from hippocampal terminals is mediated by N- and Q- but not L-type channels (Parfitt and Madison, 1993; Wheeler et al, 1994; Reuter, 1995).

Using the 30-stimulus destaining protocol described above, we next examined whether PDBu was able to increase evoked release after calcium channel blockade. The resulting data are shown in Figure $7 b$. Although absolute staining was reduced after treatment with $\omega$-CTx-GVIA and $\omega$-Aga-IVA, potentiation by PDBu was not significantly different from controls after treatment with either of these toxins or with nifedipine ( $p>0.05$, Kruskal-Wallis test).

Since none of these antagonists either increased or decreased the effect of PDBu on evoked release, we conclude that PDBu does not potentiate evoked release by selectively increasing calcium entry through N-, P/Q-, or L- type voltage-gated calcium channels. In view of published literature showing differential effects of phorbol esters at these channel subtypes (Stea et al., 1995), it is also unlikely that PDBu acts to increase calcium influx equally at all channel subtypes. Our data therefore indicate that PDBu does not potentiate evoked release by influencing calcium channel activity. In addition, these data indirectly suggest that $\mathrm{PDBu}$ does not influence release probability by another mechanism, because the effect of $\mathrm{PDBu}$ on evoked release was not enhanced in the presence of any of these antagonists (see Discussion).

Data derived using the 30 -stimulus staining protocol were more complex than those derived using the destaining protocol. Nifedipine reduced the median effect of PDBu from 109 to $68 \%(p<$ 0.0001 , Mann-Whitney rank sum test), a 38\% inhibition of the effect of PDBu. This is expected if nifedipine inhibits spontaneous but not evoked release and is therefore consistent with the data presented above (Figs. 5, 7b) and those of previous authors (Parfitt and Madison, 1993). In contrast, both $\omega$-CTx-GVIA and $\omega$-AgaIVA significantly enhanced the effect of PDBu (each $p<0.0001$, Mann-Whitney rank sum test). This probably reflects strong inhibition of evoked release by these toxins; after toxin treatment, a larger proportion of the remaining release (both before and after PDBu treatment) will result from spontaneous release. Since spontaneous release is much more strongly potentiated by $\mathrm{PDBu}$ than evoked release, the percentage increase in release after phorbol ester treatment should be greater after treatment with $\mathrm{N}$ - or Q-type channel antagonists. 
(a)

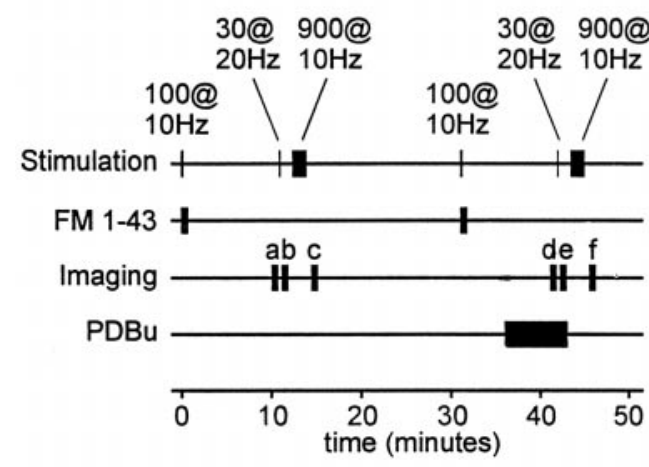

(b)

Figure 6. $\mathrm{PDBu}$ potentiates evoked release. $a$, Summary of the destaining protocol used to estimate the proportion of vesicles in the readily releasable pool. Note that $\mathrm{PDBu}$ was applied between staining and destaining steps during the second trial. $b$, Scatter plot comparing release before and after PDBu treatment at individual fluorescent puncta. Values represent the percentage of fluorescence staining released by 30 stimuli at $20 \mathrm{~Hz}$ during subsequent trials. $c$, Data represented as a frequency histogram. $d$, Frequency histogram showing the enhancement of release by phorbol ester. (c)

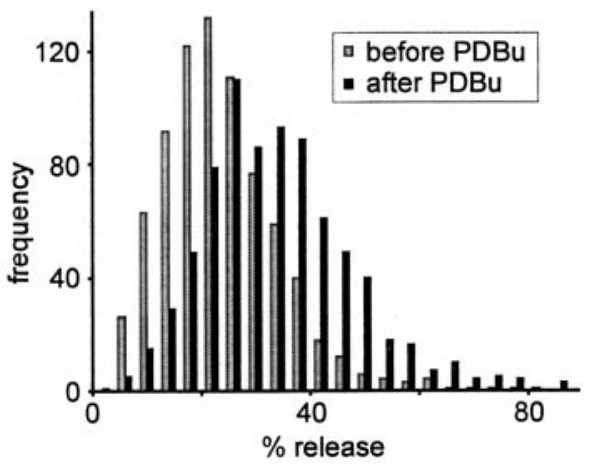

(d)

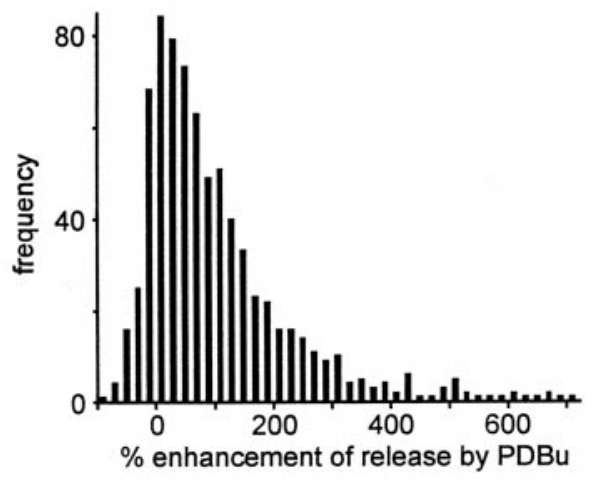

From these data we conclude that phorbol esters influence evoked and spontaneous release by different mechanisms. Potentiation of evoked release probably results from a recruiting effect of phorbol esters, whereby vesicles from the reserve pool are transferred to the readily releasable pool. In contrast, the effect on spontaneous release reflects an increase in release probability involving L-type calcium channels.

\section{DISCUSSION}

Previous publications suggested that phorbols (1) increase the number of vesicles in the readily releasable pool in hippocampal neurons in dissociated culture (Stevens and Sullivan, 1998) and (2) increase L-type calcium channel activity in hippocampal slices (Parfitt and Madison, 1993). Our data indicate that both effects coexist in the same preparation, but that they produce two distinct effects: increases in evoked and spontaneous release respectively. In addition, we have demonstrated that phorbols do not supplement the recycling vesicle pool by mobilizing new vesicles, because the total number of recycling vesicles is not altered by $\mathrm{PDBu}$. We conclude that phorbols potentiate evoked release by altering the distribution of vesicles between readily releasable and reserve pools.

We observed a mean increase in evoked release of $140 \%$ after phorbol ester treatment, comparable with that reported by Stevens and Sullivan (1998), who used hypertonic solutions to induce release. This similarity is important, because we were unable to definitively discount the possibility that the observed potentiation resulted from increased release probability per vesicle. In contrast to action potential-induced release, hypertonic solutions release vesicles in a calcium-independent manner, so alterations in release probability per vesicle will have little effect on this measure of readily releasable pool size (Rosenmund and Stevens, 1996). The fact that our data show potentiation comparable with that measured using hypertonic solutions therefore suggests that the potentiation of evoked release that we observed reflects a change in the number of vesicles in the readily releasable pool rather than an increased release probability per vesicle.

Using electrophysiological techniques, Murthy and Stevens
(1999) reported that $\sim 30 \%$ of recycling vesicles were within the readily releasable pool. Unfortunately, the temporal resolution afforded by FM dye imaging techniques prevents estimation of the readily releasable pool size in the manner of electrophysiological experiments, in which one can calculate the number of vesicles released before depletion. From electrophysiological data, one would expect 30 stimuli delivered at $20 \mathrm{~Hz}$ to be sufficient to exocytose the entire readily releasable pool (Murthy and Stevens, 1998). However, in our experiments the average proportion of vesicles released by this stimulus was slightly less than $30 \%$ (see Fig. $6 c$ ). Our protocol may therefore slightly underestimate the proportion of recycling vesicles in the readily releasable pool.

The fact that 30 stimuli at $20 \mathrm{~Hz}$ release almost the entire readily releasable pool before phorbol exposure further suggests that the effects of PDBu were the result of an increase in the number of vesicles in the readily releasable pool. Since most of the readily releasable pool was released by this stimulus under control conditions, it is unlikely that a large potentiation by $\mathrm{PDBu}$ could be observed without mobilization of extra vesicles to the readily releasable pool. In view of this "ceiling" on the effect of PDBu that could result without the mobilization of additional vesicles, it is unlikely that an increase in release probability per vesicle could account for the large potentiation observed.

Furthermore, if potentiation by PDBu was limited by this ceiling effect, one might expect to see increased potentiation of evoked release after reducing release probability with calcium channel antagonists. No such effect was observed (Fig. 7b). Together these data suggest that phorbols enhance evoked release by altering the proportion of recycling vesicles in the readily releasable pool rather than increasing release probability per vesicle.

\section{Destaining protocol as a measure of evoked release}

Using both direct and indirect protocols, we concluded that PDBu treatment enhances evoked release by $\sim 50 \%$. Changes in spontaneous release rates may have influenced our measurements of evoked release given the pronounced effect of $\mathrm{PDBu}$ on spontaneous release. However, spontaneous loss of fluorescence was extremely slow, even after PDBu treatment (data not shown). Con- 
(a) Effects of antagonists in absence of PDBu.

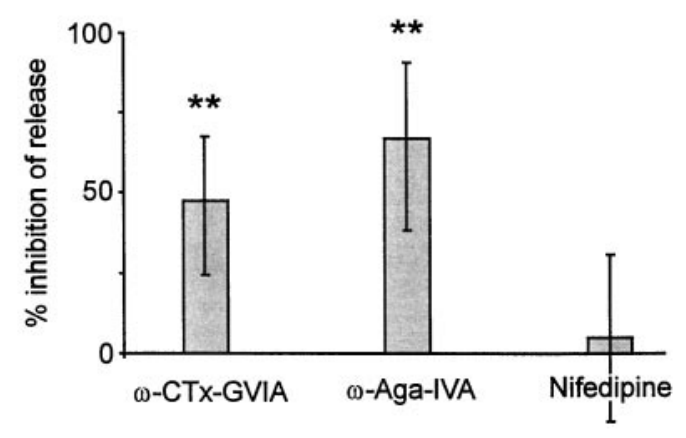

(b) Effect of PDBu on evoked release

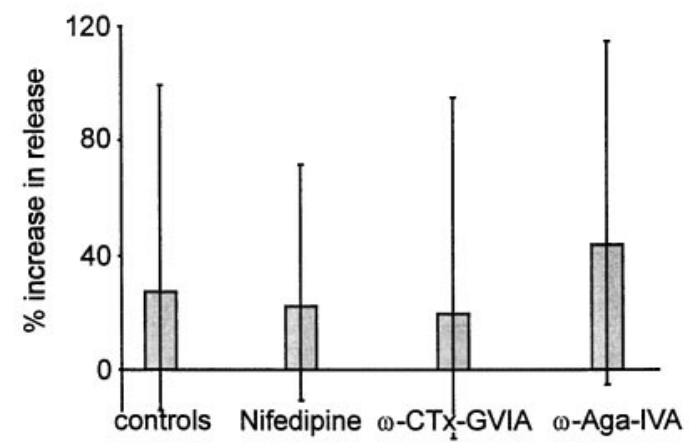

(c) Effect of PDBu on evoked + spontaneous release

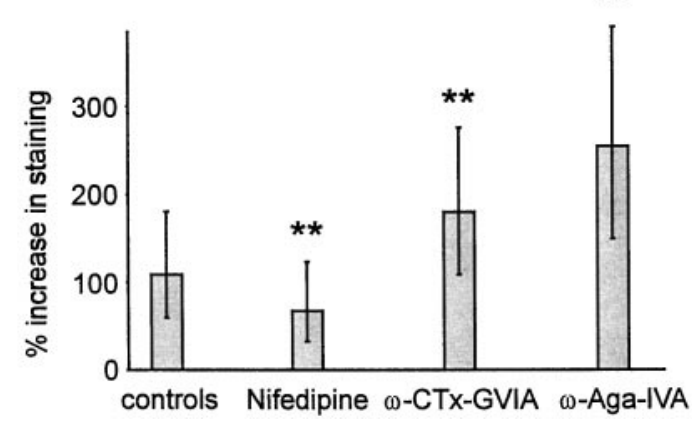

Figure 7. Effects of calcium channel antagonists. $a$, Effects of calcium channel antagonists on evoked release (no PDBu). Data were derived using a 60 -stimulus $(10 \mathrm{~Hz})$ destaining protocol, after staining using a 100 stimulus train at $10 \mathrm{~Hz}$. Preparations were subjected to two trials, the first a control and the second after or during application of antagonist. $\omega$-CTxGVIA $(1 \mu \mathrm{M})$ was applied for $10 \mathrm{~min}$ after the second staining step. $\omega$-Aga-IVA $(500 \mathrm{nM})$ and nifedipine $(10 \mu \mathrm{M})$ were each applied for $5 \mathrm{~min}$ before the second destaining step and remained in the perfusion chamber during destaining. $n$ : nifedipine, 86; $\omega$-CTx-GVIA, 344; $\omega$-Aga-IVA, 100. $b$, Calcium channel antagonists failed to attenuate the effect of PDBu on evoked release measured using a 30-stimulus destaining assay (as in Fig. $6 a)$. Data are derived from two consecutive trials, the first before and the second after PDBu exposure (as in Fig. 6). Controls were not treated with calcium channel antagonists $(n=391)$. Nifedipine-treated preparations $(n=279)$ were perfused throughout with $10 \mu \mathrm{M}$ nifedipine, beginning 5 min before the start of the first trial. $\omega$-CTx-GVIA effects were examined by pretreating the preparation with $1 \mu \mathrm{M} \omega$-CTx-GVIA for 10 min before the start of the first trial $(n=194)$. $\omega$-Aga-IVA (500 nM) was applied for 5 min before each staining or destaining stimulus and was also present in the perfusion chamber throughout all destaining stimulus trains $(n=150)$. $c$, Influence of antagonists on PDBu-induced potentiation using a 30 -stimulus staining protocol (as in Fig. 2a). Antagonists were applied as described above (controls, $n=531 ; 10 \mu \mathrm{M}$ nifedipine perfused throughout, $n=326$; $1 \mu \mathrm{M} \omega$-CTx-GVIA by pretreatment for $10 \mathrm{~min}, n=105 ; 500 \mathrm{nM} \omega$-AgaIVA by pretreatment for $5 \mathrm{~min}$ and perfused throughout stimulation, $n=$ 106). Throughout parts $a-c$, column heights represent medians, and error bars represent the quartile $(25-75 \%)$ ranges of each distribution. sequently, intensities of puncta in the stained condition were reduced only $\sim 9 \%$ (median) by a 5 min treatment with $\mathrm{PDBu}$ (data not shown). This probably reflects a very low rate of spontaneous release before $\mathrm{PDBu}$ application, such that even pronounced potentiation of spontaneous release by $\mathrm{PDBu}$ produced only modest rates of spontaneous destaining. Although the destaining protocol is therefore not entirely unaffected by spontaneous release, even large changes in spontaneous release rates do not influence the data on evoked release derived in this manner. Furthermore, the effects of spontaneous release on data derived with the evoked release protocol would have been mostly eliminated by the analysis method used, in which the effects of the destaining stimulus were expressed as a proportion of the fluorescence intensity in the stained condition.

Although an experimental approach that definitively separated evoked and spontaneous release was not available, it is therefore likely that the destaining protocol used (Fig. 6) separated evoked and spontaneous release sufficiently to yield accurate data describing the effect of PDBu on evoked release. Consequently, destaining measurements (Fig. 6) and calculations based on staining protocols (Figs. 2, 4, 5) yielded similar estimates for the effect of PDBu on evoked release (median potentiations of 49 and 52\%, respectively).

\section{Molecular mechanism of action of phorbol esters}

The molecular targets of $\mathrm{PDBu}$ are unknown, but it has been suggested that phorbols increase the size of the readily releasable pool through actions at two steps in the secretion pathway (Bittner and Holz, 1993). This may reflect interactions with both protein kinase $\mathrm{C}$ and another presynaptic phorbol ester-activated protein such as Munc 13-1 (Goda et al., 1996; Betz et al., 1998). In murine hippocampus, Munc 13-1 is thought to be involved in a calciumindependent priming step that gates entry into the readily releasable pool (Augustin et al., 1999). At the Calyx of Held, PDBu potentiates release through actions on both protein kinase $\mathrm{C}$ and Munc 13-1 (Hori et al., 1999). In light of these reports, our data suggest that PDBu might act through three mechanisms; a protein kinase C-mediated increase in vesicle docking, a Munc 13-1mediated increase in vesicle priming, and an action on L-type calcium channels (probably through protein kinase C).

Serial reconstruction of hippocampal synapses from electron micrographs has revealed that on average no more than half the docking sites at an active zone are occupied (Harris and Sultan, 1995; Schikorski and Stevens, 1997). It is possible, therefore, that active zones could accommodate an increased number of docked vesicles. Increased docking (presumably via protein kinase $\mathrm{C}$ activation) is therefore one potential mechanism by which phorbol esters might increase the size of the readily releasable pool. The molecular targets by which protein kinase $\mathrm{C}$ may induce vesicle docking are unknown, but there are numerous presynaptic proteins that are phosphorylated after activation of protein kinase $\mathrm{C}$. These include cytoskeletal proteins that could influence vesicle trafficking (Vitale et al., 1995; Ryan, 1999) as well as docking/release proteins such as VAMP, SNAP25, Munc 18, and the synapsins (Browning and Dudek, 1992; Turner et al, 1999).

Other potential synaptic effects of phorbol esters have been identified, including effects on potassium channels, on calcium clearance, and on the release machinery itself. Although we have not specifically addressed these effects, it seems unlikely that these mechanisms are responsible for our observations, because all three relate to calcium metabolism. Were alterations in calcium metabolism a factor underlying the effects of PDBu, one would expect inhibition of calcium influx to influence the effects of PDBu. Since calcium channel antagonists did not alter the potentiating effect of $\mathrm{PDBu}$ on evoked release, it is unlikely that $\mathrm{PDBu}$ acts through these alternative routes at hippocampal synapses.

\section{Model to account for the effects of phorbol esters}

The fact that phorbol esters influence spontaneous release through L-type calcium channel activation is surprising in view of the fact that L-type calcium channels do not mediate presynaptic release 
from hippocampal terminals (Parfitt and Madison, 1993; Wheeler et al., 1994). Such channels are, however, distributed throughout hippocampal neurons and influence the resting intracellular calcium concentration through tonic activity (Magee et al., 1996). The effect of phorbol esters on spontaneous release may therefore reflect a modest rise in intracellular calcium concentration throughout the neuron rather than a localized effect within the presynaptic terminal.

We conclude that phorbol esters influence release from hippocampal terminals by several mechanisms. Spontaneous release is increased, probably through an extrasynaptic effect of phorbol esters on L-type calcium channels. This does not influence evoked release. In addition, phorbol esters probably activate both protein kinase $\mathrm{C}$ and Munc 13-1, causing increased vesicle docking and priming, respectively. Together these effects on docking and priming are observed functionally as an increased number of vesicles in the readily releasable pool. We have also shown that these effects do not involve mobilization of new vesicles into the recycling vesicle pool. Hence phorbol esters must induce redistribution of vesicles from the reserve to the readily releasable pool. Our data therefore identify an increase in the proportion of recycling vesicles in the readily releasable pool as the functional mechanism by which phorbol esters potentiate release at hippocampal synapses. In addition to clarifying the mechanism of action of phorbol esters, our data indicate that phorbol ester treatment may be used to selectively probe the functions of different presynaptic vesicle pools.

\section{REFERENCES}

Augustin I, Rosenmund C, Südhof TC, Brose N (1999) Munc 13-1 is essential for fusion competence of glutamatergic synaptic vesicles. Nature 400:457-461.

Betz WJ, Bewick GS (1992) Optical analysis of synaptic vesicle recycling at the frog neuromuscular junction. Science 255:200-203.

Betz WJ, Mao F, Smith CB (1996) Imaging exocytosis and endocytosis. Curr Opin Neurobiol 6:365-371.

Betz A, Ashery U, Rickmann M, Augustin I, Neher E, Südhof TC, Rettig J, Brose N (1998) Munc 13-1 is a presynaptic phorbol ester receptor that enhances neurotransmitter release. Neuron 21:123-136.

Bittner MA, Holz RW (1993) Protein kinase C and clostridial neurotoxins affect discrete and related steps in the secretory pathway. Cell Mol Neurobiol 13:649-664.

Browning MD, Dudek EM (1992) Activators of protein kinase C increase the phosphorylation of the synapsins at sites phosphorylated by cAMPdependent and $\mathrm{Ca}^{2+} /$ calmodulin-dependent protein kinases in the rat hippocampal slice. Synapse 10:62-70.

Gillis KD, Mößner R, Neher E (1996) Protein kinase C enhances exocytosis from chromaffin cells by increasing the size of the readily releasable pool of secretory granules. Neuron 16:1209-1220.

Goda Y, Stevens CF, Tonegawa S (1996) Phorbol ester effects at hippocampal synapses act independently of the gamma isoform of PKC. Learn Mem 3:182-187.

Harris KM, Sultan P (1995) Variation in the number, location and size of synaptic vesicles provides an anatomical basis for the nonuniform probability of release at hippocampal CA1 synapses. Neuropharmacology 34:1387-1395.

Henkel AW, Lübke J, Betz WJ (1996) FM1-43 dye ultrastructural local- ization in and release from frog motor nerve terminals. Proc Natl Acad Sci USA 93:1918-1923.

Hoffman DA, Johnston D (1998) Downregulation of transient $\mathrm{K}^{+}$channels in dendrites of hippocampal CA1 pyramidal neurons by activation of PKA and PKC. J Neurosci 18:3521-3528.

Hori T, Takai Y, Takahashi T (1999) Presynaptic mechanism for phorbol ester-induced synaptic potentiation. J Neurosci 19:7262-7267.

Lin LF, Kao LS, Westhead EW (1994) Agents that promote protein phosphorylation inhibit the activity of the $\mathrm{Na}^{+} / \mathrm{Ca}^{2+}$ exchanger and prolong $\mathrm{Ca}^{2+}$ transients in bovine chromaffin cells. J Neurochem 63:1941-1947.

Magee JC, Avery RB, Christie BR, Johnston D (1996) Dihydropyridinesensitive, voltage-gated $\mathrm{Ca}^{2+}$ channels contribute to the resting intracellular $\mathrm{Ca}^{2+}$ concentration of hippocampal CA1 pyramidal neurons. J Neurophysiol 76:3460-3470.

Malenka RC, Madison DV, Nicoll RA (1996) Potentiation of synaptic transmission in the hippocampus by phorbol esters. Nature 321:175-177.

Murthy VN (1999) Optical detection of synaptic vesicle exocytosis and endocytosis. Curr Opin Neurobiol 9:314-320.

Murthy VN, Stevens CF (1998) Synaptic vesicles retain their identity through the endocytic cycle. Nature 392:497-500.

Murthy VN, Stevens CF (1999). Reversal of synaptic vesicle docking at central synapses. Nat Neurosci 2:503-507.

Parfitt KD, Madison DV (1993) Phorbol esters enhance synaptic transmission by a presynaptic, calcium-dependent mechanism in rat hippocampus. J Physiol (Lond) 471:245-268.

Redman RS, Searl TJ, Hirsh JK, Silinski EM (1997) Opposing effects of phorbol esters on transmitter release and calcium currents at frog motor nerve endings. J Physiol (Lond) 501.1:41-48.

Reuter H (1995) Measurements of exocytosis from single presynaptic nerve terminals reveal heterogeneous inhibition by $\mathrm{Ca}^{2+}$ channel blockers. Neuron 14:773-779.

Rosenmund C, Stevens CF (1996) Definition of the readily releasable pool of vesicles at hippocampal synapses. Neuron 16:1197-1207.

Ryan TA (1999) Inhibitors of myosin light chain kinase block synaptic vesicle pool mobilization during action potential firing. J Neurosci 19:1317-1323.

Ryan TA, Smith SJ (1995) Vesicle pool mobilization during action potential firing at hippocampal synapses. Neuron 14:983-989.

Ryan TA, Reuter H, Wendland B, Schweizer FE, Tsien RW, Smith SJ (1993) The kinetics of synaptic vesicle recycling measured at single presynaptic boutons. Neuron 11:713-724.

Ryan TA, Smith SJ, Reuter H (1996) The timing of synaptic vesicle endocytosis. Proc Natl Acad Sci USA 93:5567-5571.

Ryan TA, Reuter H, Smith SJ (1997) Optical detection of a quantal presynaptic membrane turnover. Nature 388:478-482.

Schikorski T, Stevens CF (1997) Quantitative ultrastructural analysis of hippocampal excitatory synapses. J Neurosci 17:5858-5867.

Stea A, Soong TW, Snutch TP (1995) Determinants of PKC-dependent modulation of a family of neuronal calcium channels. Neuron 15:929-940.

Stevens CF, Sullivan JM (1998) Regulation of the readily releasable vesicle pool by protein kinase C. Neuron 21:885-893.

Turner KM, Burgogne RD, Morgan A (1999) Protein phosphorylation and the regulation of synaptic membrane traffic. Trends Neurosci 22:459-464.

Vitale ML, Seward EP, Trifaró J-M (1995) Chromaffin cell cortical actin network dynamics control the size of the release-ready vesicle pool and the initial rate of exocytosis. Neuron 14:353-363.

Wheeler DB, Randall A, Tsien RW (1994) Roles of N-type and Q-type $\mathrm{Ca}^{2+}$ channels in supporting hippocampal synaptic transmission. Science 264:107-111. 